\title{
Evolution de la position du sujet pronominal en français médiéval : une approche sémantico- pragmatique
}

\author{
Sophie Prévost \\ Lattice, UMR 8094 (CNRS/ENS Paris) \\ sophie.prevost@ens.fr
}

\section{Introduction}

La syntaxe du sujet est fortement contrainte en français moderne : le sujet est toujours exprimé (sauf cas de coordination immédiate) et il se trouve majoritairement en position préverbale. Les sujets nominaux ne se rencontrent en position postverbale que dans certains contextes informationnels (voir entre autres Fuchs 2006, Lahousse 2003 et Marandin 2003 pour des approches complémentaires). En ce qui concerne les sujets pronominaux (pronoms personnels) leur inversion a toujours été beaucoup plus rare que celle des sujets nominaux et elle est corrélée, en français moderne, à la présence en position initiale d'adverbes épistémiques ou argumentatifs (voir Guimier 1997), comme en (1) :

(1) Paul est très fatigué en ce moment : aussi a-t-il décidé de renoncer à son voyage ; peut-être changera-t-il d'avis dans quelques jours. (énoncé construit).

S'inscrivant dans un vaste projet en cours portant sur l'évolution de la syntaxe du sujet pronominal en français dans les propositions déclaratives, cet article vise à en présenter les premiers résultats. On rappellera tout d'abord brièvement les grands traits de la syntaxe du sujet en ancien français, puis on s'attachera plus spécifiquement à rendre compte de l'alternance positionnelle du sujet pronominal dans les propositions déclaratives, et l'on finira en suggérant de possibles explications à la rigidification de l'ordre Sujet-Verbe.

\section{Caractéristiques de la syntaxe du sujet en ancien français}

\subsection{Des caractéristiques romanes et germaniques}

D'une manière générale, l'ordre des mots était plus souple en ancien français qu'il ne l'est en français moderne, au sens où il n'était pas régi par les fonctions syntaxiques : l'objet nominal pouvait occuper la position préverbale et le sujet se trouver après le verbe. En ce qui concerne plus précisément le sujet, l'ancien français présente les caractéristiques des langues romanes, dans la mesure où il autorise la nonexpression du sujet (c'est une langue "pro-drop ») et le type d'inversion du sujet qui lui est associée, l'inversion « romane» : le sujet nominal suit l'ensemble des formes verbales (ainsi que la négation pas), comme en $(2 \mathrm{a}-\mathrm{b})$ :
(2a) Tout einsint ont anonciee li hermite et li saint home vostre venue plus a de vint anz (Queste del Saint Graal, vers 1220)
(2b) bele buce, bel vis, bele faiture,
Cum est mudede vostra bela figure ! (Vie Saint Alexis, 1050)

L'ancien français présente cependant aussi des caractéristiques des langues germaniques; il s'agit d'une part de la contrainte du verbe en seconde position ( «contrainte V2»), avec un élément tonique en première position. Assez stricte, cette contrainte n'est cependant pas absolue, puisque l'on rencontre des occurrences de verbe en $1^{\text {ère }}$ position comme en $(3 a-b)^{1}$ ou en $3^{\text {ème }}$ position, comme en $(4 a-c)$ :

(3a) Plurent Franceis pur pitet de Rollant (Chanson de Roland, vers 1100)

(3b) Ot le Gillelmes, s'en a un ris gité (Le Charroi de Nimes, $12^{\text {ème }}$ siècle) 
(4b) Veez m'espee, ki est e bone e lunge :

A Durendal jo la metrai encuntre (Roland)

(4c) Par cele foi que je vos doi /

Se cel anel de vostre doi

Ne m'envoiez, si que jel voie,

Rien qu'il deïst ge ne croiroie (Beroul, fin $12^{\text {ème }}$ )

(4d) «...et gardez qu' il ne soit a nul home mortel conté que vos l'aiez veü en ceste voie, ne ge endroit moi n'en parlerai ja . » (Mort Artu, 1230)

Il s'agit d'autre part du type d'inversion qui lui est associée, l'inversion dite germanique, dans laquelle le sujet nominal ou pronominal suit immédiatement la forme finie du verbe, et précède les autres formes verbales (telles que les participes et les infinitifs) :

(5a) Si a li rois einsi atendu des le tens Josephe jusqu'à ceste hore (Queste del Saint Graal, vers 1220)

(5b) ... et einsi furent il destruit par l'anemi et par son amonestement (idem)

Pour les sujets nominaux l'inversion romane semble moins fréquente en ancien français que l'inversion germanique, mais elle est néanmoins attestée dès les premiers textes (comme en témoigne (2b)). Elle va peu à peu s'imposer et elle est aujourd'hui la seule possible.

\subsection{L'expression du sujet}

La non-expression du sujet était fréquente en ancien français, elle pouvait atteindre plus de $50 \%$ des cas dans les propositions déclaratives (Chanson de Roland : 74\%; Queste des Saint Graal : 51\%; voir Marchello-Nizia 1995) ${ }^{2}$. Le sujet était omis dès que le référent était facilement identifiable, alors que, exprimé, il signalait une discontinuité thématique (un changement de temps ou de référent par exemple) ou une opposition, ou une insistance particulière. Cela n'était cependant pas une règle systématique : on rencontre parfois des sujets pronominaux associés à une continuité thématique (en particulier après une subordonnée temporelle). La variation peut se produire d'un texte à l'autre, mais aussi, plus étonnamment, au sein d'un même texte ${ }^{3}$, sans que l'on puisse détecter des différences notables entre les variables, comme c'est le cas en (6) et (7) dans La Mort Artu :

(6) Quant Agravains se fu aperceüz de la reïne et de Lancelot, il en fu liez durement et plus por le domage que il cuida que Lancelos en eüst que por le roi vengier de sa honte (la mort Artu,1230)

(7) Et quant Agravains sot que Boorz s'en aloit et li chevalier avec lui et que Lancelos remanoit, si pensa tantost que c'estoit por la reïne ou il vouloit avenir, quant li rois s'en seroit alez. (idem)

La continuité thématique ne suffit donc pas à expliquer l'omission du sujet, et seule une étude détaillée sur un corpus large permettra d'en déterminer les modalités exactes. Nous laissons ici cette question de côté $^{4}$, mais souhaitons néanmoins dire quelques mots de la « nature » du sujet non-exprimé. On considère généralement que les sujets omis correspondent à des sujets pronominaux (pour des raisons référentielles évidentes), mais il n'y a en revanche pas de consensus pour ce qui est de leur position potentielle. Il a été affirmé, dans des approches assez différentes (voir en particulier Foulet 1965, Skårup 1975, Vance 1997, Buridant 2000), que les sujets omis seraient équivalents à des pronoms postverbaux 5 . On avance deux arguments principaux pour cela; le premier, syntaxique, est lié à la contrainte du verbe en seconde position : du fait que l'on observe souvent des séquences $\mathrm{CV}(\mathrm{X})$, on en conclut que, s'il avait été exprimé, le pronom aurait suivi le verbe. Or on rencontre aussi des séquences CSpV (rarement il est vrai 
en ancien français $)^{6}$, comme en (4b-c) ci-dessus et en (8) ci-après, ainsi que des séquences SpXV (voir (4d)) :

(8) Sire nos volons que vos aiez vostre conseil; et devant vostre conseil nos vos dirons ce que nostre seignor vos mandent (Villehardouin, Conqueste de Constantinople, vers 1200)

L'autre argument est de nature pragmatique : les contextes des sujets omis et des sujets postverbaux seraient équivalents. Or, précisément, ce n'est généralement pas le cas: les sujets pronominaux postverbaux sont rares, comme on va le voir, et les constructions sont donc marquées d'un point de vue quantitatif. Elles le sont aussi qualitativement ${ }^{7}$.

C'est pourquoi il nous semble préférable de considérer qu'il existe trois types de sujets pronominaux : ceux qui sont omis, ceux qui sont préverbaux, et enfin ceux qui sont postverbaux, les premiers étant les plus fréquents et les derniers les plus rares.

\subsection{La position du sujet pronominal}

Rappelons qu'il existe des différences phonétiques et syntaxiques importantes selon la position du sujet : en position préverbale le pronom peut porter l'accent et être disjoint, comme en (9) :

\section{(9) Et ele tant le conforta}

[....] Que ele en santé le remist (P. de Rémi, Jehan et Blonde, vers 1230)

Il peut aussi être coordonnée et déterminé comme en (10a) et (10b):

(10b) Jou et mi homme nous voulons vengier d'aus (Clari, la Conqueste de Constantinople, après 1205)

(10b) Et je, ki ai apris autres salus de cevaliers et autres acointements, m'en retournerai au roiaume de la Petite Bretaigne (Tristan en prose, après 1240)

Le sujets postverbaux sont en revanche toujours conjoints, ils ne peuvent être séparés du verbe que s'ils appartiennent à un groupe, comme en (11):

\section{(11) or avront garnemenz il e si cumpaignon}

Dans le modèle positionnel de Skårup (1975), le sujet pronominal préverbal se trouve dans la zone préverbale, comme le sujet nominal, tandis que le sujet pronominal postverbal se trouve dans la zone verbale, sauf s'il est accompagné d'un autre élément, comme en (11). Dans ce cas, il se trouve dans la zone postverbale (comme le sujet nominal qui, lui, y est systématiquement) ${ }^{8}$.

Le pronom s'est cliticisé à partir du $13^{\text {ème }}$ ou du $14^{\text {ème }}$ siècle ${ }^{9}$ : même en position préverbale il ne lui a plus été possible d'être séparé du verbe ni de porter l'accent.

Pendant longtemps on a justifié la souplesse de l'ordre des mots en ancien français par l'existence d'une déclinaison associée à la contrainte V2. Dès lors qu'un élément X se trouvait en tête, le sujet était postposé au verbe. La chute de la déclinaison et le recul de V2 auraient entraîné la fixation de l'ordre des mots. L'explication est néanmoins insuffisante : on trouve en ancien français des occurrences de verbe en première et en troisième position (voir $(3 a-b)$ et $(4 a-d)$ ), et la déclinaison est déficiente dès le $13^{\text {ème }}$ siècle. A la suite des travaux de Vennemann (1976) et de Combettes (1988), l'explication a été complétée par un principe fonctionnel : l'ordre des mots était régi par un principe informationnel (formulé en termes de « topique-commentaire » ou de « thème-rhème » selon les approches) : le sujet est postposé, soit quand il n'est pas le topique, soit quand il est porteur d'une charge informative élevée. Si le recours au principe informationnel est assez convaincant pour les sujets nominaux ${ }^{10}$, il est en revanche beaucoup moins évident pour les sujets pronominaux, au moins pour le pronom de $3^{\text {ème }}$ (et $6^{\text {ème }}$ ) personne, le plus fréquent. Il correspond en effet à un topique par excellence, et il est porteur d'une faible charge informative. Cela explique peut-être d'ailleurs la relative rareté de sa postposition comparée à celle des sujets nominaux. Comment donc expliquer les structures VS ? Y-a-t-il des régularités, une évolution ? C'est à ces questions 
que nous allons essayer de répondre, en montrant que seule la prise en compte de l'énoncé dans son ensemble, et des éléments initiaux en particulier, permet d'apporter des éléments de réponse. Nous nous en tiendrons ici aux occurrences des pronoms de $3^{\text {ème }}$ et $6^{\text {ème }}$ personnes, remettant à une étude ultérieure celles de $1^{\text {èr }}$ personne, qui mettent en jeu une dimension énonciative spécifique ${ }^{11}$.

\section{Corpus et quantification des données}

Le corpus d'étude est composé de 2 textes de la fin du $13^{\text {ème }}$ et du début du $14^{\text {ème}}$ : Tristan en prose, abr. Tristan (graphiste picard) et La vie de Saint Louis de Joinville, abr. Joinville (Champagne), et de deux textes de la fin du $14^{\text {ème }}$ et du début du $15^{\text {ème }}$ siècle : Les Quinze Joyes de mariage, abr. QJM (région ouest, peut-être le Poitou), et le livre 1 des Chroniques de Froissart, abr. Froissart (picard).

Les paramètres de variation entre textes portent donc sur la date et le genre (mémoires/chroniques et roman/nouvelles), et sur la région, dont la pertinence, au regard de ce point précis de syntaxe, n'est pas assurée. Pour chaque texte nous avons travaillé sur un échantillon d'environ 35000 mots, ce qui correspond à l'intégralité des Quinze Joyes de mariage, et au début des œuvres pour les autres textes. Ces données ont été complétées par quelques remarques sur des textes plus anciens : la chanson de Roland (1100), abr. Roland, Tristan de Beroul (fin 12 ème), abr. Beroul, et La mort Artu (vers 1230), abr. Artu, ainsi que sur des textes un peu plus tardifs (fin $15^{\text {ème }}$ ): La Chronique d'Enguerrand de Monstrelet, abr. Monstrelet, Les cent nouvelles nouvelles, abr. CNN, Le Roman de Jehan de Paris, abr. Jehan, et le livre 1 des Mémoires de Commynes, abr. Commynes ${ }^{12}$.

La démarche a consisté à comparer les structures $\mathrm{SpV}$ et VSp en portant une attention spécifique aux éléments initiaux. Rappelons que les relevés ont été effectués dans les seules propositions déclaratives, indépendantes et principales (suppression des incises: dit-il, fet-ele... qui représentent 41 cas sur 132 dans Tristan)

Le tableau 1 ci-dessous présente les résultats des relevés (les chiffres en italiques gras correspondent au pourcentage du type d'énoncés sur le total des énoncés à sujet pronominal (dernière colonne).

\begin{tabular}{|c|c|c|c|c|c|}
\hline & \multicolumn{2}{|c|}{ Enoncés V-Sp } & \multicolumn{2}{|c|}{ Enoncés $\mathrm{Sp}-\mathrm{V}$} & Total Sp \\
\hline Roland -1100 & 24 & 29.3 & 58 & 70.7 & 82 \\
\hline Beroul - fin $12^{\text {ème }}$ & 21 & 16.8 & 104 & 83.2 & 125 \\
\hline Artu $-1^{e r}$ tiers $13^{\text {ème }}$ & 39 & 16.5 & 198 & 83.5 & 237 \\
\hline Tristan - fin $13^{\text {ème }}$ & 91 & 21.6 & 330 & 78.4 & 421 \\
\hline Joinville - début $14^{\grave{e} m e}$ & 19 & 7.1 & 248 & 92.9 & 267 \\
\hline Froissart - fin $14^{\grave{e ̀ m e}}$ & 38 & 18 & 173 & 82 & 211 \\
\hline QJM - début $15^{\text {ème }}$ & 25 & 7.2 & 323 & 92.8 & 348 \\
\hline Monstrelet - mil $15^{\text {ème }}$ & 1 & 1.3 & 75 & 98.7 & 76 \\
\hline CNN - mil $15^{\text {ème }}$ & 14 & 7.7 & 167 & 92.3 & 181 \\
\hline Commynes - fin $15^{\text {ème }}$ & 7 & 6.9 & 95 & 93.1 & 102 \\
\hline Jehan - fin $15^{\text {ème }}$ & 11 & 7.2 & 142 & 92.8 & 153 \\
\hline
\end{tabular}

Tableau 1 : Répartition des énoncés à sujet préverbal et postverbal. 
Le premier constat est la grande disparité des chiffres. D'une part le nombre d'occurrences de sujets pronominaux de $3^{\text {ème }}$ et $6^{\text {eme }}$ personnes (désormais P3) varie du simple au double (entre Froissart et Tristan) et d'autre part le pourcentage d'inversion a un rapport de 1 à 3 (Joinville et QJM par opposition à Tristan). Au premier regard on ne voit pas se dégager de tendance nette, mais l'on observe en revanche des affinités surprenantes, avec d'un côté Froissart et Tristan, et QJM et Joinville de l'autre, alors qu'il s'agit de textes appartenant à des époques et à des genres différents (le seul point commun entre Tristan et Froissart est qu'ils présentent tous les deux des traits picards).

Toutefois, si l'on met Froissart de côté, et que l'on regarde les textes qui précèdent et qui suivent notre corpus noyau, on constate que les pourcentages d'inversion sont nettement plus élevés dans Roland, Beroul, Artu et Tristan, et que Joinville semble marquer un tournant : les pourcentages à partir de ce texte avoisinnent 7\%, avec néanmoins deux exceptions notables : Froissart (18\%) et Monstrelet (1.3\%). Il semble même y avoir un premier tournant au $12^{\text {ème }}$ siècle (on passe de $29.3 \%$ à $16.8 \%$ ), mais il faudrait examiner d'autres textes contemporains ou antérieurs à Roland pour confirmer cette impression.

\section{Analyse des données}

\subsection{Sous l'éparpillement : une relative complémentarité}

A première vue, il n'est pas facile d'opposer les énoncés VSp et $\mathrm{SpV}$, et cela dans tous les textes ${ }^{13}$. Dans les deux cas, la proposition peut être en toute position (début de phrase, ou coordonnée, ou bien encore derrière une subordonnée). Par ailleurs, pour ce qui est de la mention du sujet dans la proposition précédente (quelle que soit la nature de celle-ci : indépendante ou subordonnée), on rencontre tous les cas de figure : son expression peut être nominale comme en (12) et (13), pronominale comme en (14) et (15), en fonction sujet ou complément, mais l'on rencontre aussi des cas de non-expression, généralement en fonction sujet, ou bien encore, bien que plus rarement, l'absence, soit que le référent du sujet ait été mentionné dans une proposition plus lointaine, soit qu'il soit inférable.

(12) Ensi conmence la mellee grans et merveilleuse des .IIII. chevaliers du castel encontre Lanselot. II l'asaillent mout aigrement de toutes pars...(Tristan)

(13) Et a cele bataille frere Guillaume, le mestre du Temple, perdi l'un des yex, et l'autre avoit il perdu le jour de quaresme pernant < mardi gras $>$ (Joinville)

(14) ...mes elle a bien a faire ciens et elle est tourjours par chemins (QJM)

(15) Et est lor terre plus plainne de riçoisses et de tous biens, qant $\boldsymbol{i l}$ ont la gerre, que en temps de paix. Et en cela sont il né et obstiné, ne nuls ne lor poroit faire entendant le contraire (Froissart)

Toutefois, alors que la succession d'énoncés $\mathrm{SpV}$ est courante, la succession d'énoncés VSp est peu fréquente : les rares occurrences rencontrées se trouvent uniquement dans Artu (1 seule) et dans Tristan (6 occurrences) :

(16) Se ce est Lanselos, li boins cevaliers, il li laissera tout maintenant ceste bataille, car encontre lui ne se porroit il pas au daerrain parfurnir, ce set il bien certainnement. Et se ce est mesire Tristrans, li boins cevaliers de Cornuaille, a celui ne se veut il prendre ne pour mort ne pour vie, car de combatre encontre monsigneur Tristan ne porroit il avoir fors que la mort. Encontre nul de ces deus ne se combatroit il en nule maniere du monde, pour k'il le seüst, mais a tous autres cevaliers il se combateroit hardiement. (Tristan)

Sans doute faudrait-il affiner l'examen des modalités de l'expression du sujet dans le contexte antérieur, en dépassant les limites de la seule proposition précédente. Mais il faut aussi considérer l'énoncé dans son ensemble, c'est-à-dire la relation qu'il entretient avec le contexte précédent, relation qui est en partie 
exprimée par les éléments initiaux. L'examen de ces derniers laisse en effet apparaître certaines tendances, qui, de prime abord, semblent davantage concerner les énoncés SpV.

On observe en effet pour ces derniers la présence récurrente en tête de certains éléments, cela dans tous les textes, bien que dans des proportions variables. Trois structures en particulier apparaissent régulièrement. Il s'agit d'une part de la présence du sujet en position initiale :

(17) Lors les commeres entrent. Elles desjunent, elles dignent, elles menjent a raassie : maintenent boivent au lit de la commere, maintenant a la cuve, et confondent des biens et du vin plus qu' il n'en entreroit en une bote $(Q J M)$ :

Il s'agit d'autre part de la présence d'une conjonction de coordination (seule) : et, car $^{14}$ ou mais (18 et 19), ou de celle d'une subordonnée temporelle (exprimant le plus souvent la concomitance ou la postériorité immédiate), précédée ou non d'une conjonction de coordination (20) :

(18) Le roy respondi que il en pooient faire leur volenté, car il amoit miex mourir bon crestien que ce qu'il vesquist ou courrous Dieu et sa Mere et de ses saints (Joinville)

(19) Et qant chils bons rois Edouwars fu trespassés, ses fils, nonmés aussi Edouwars, fu rois, mais il n'ensievi pas ne en riens la vaillance dou roi son pere.(Froissart)

(20) Quant li vallés voit et connoist que li cevaliers du pont li retient en tel maniere le passage, il crie a son signeur:...(Tristan)

Nous présentons dans le tableau 2 ci-dessous la répartition en pourcentages des différents cas:

\begin{tabular}{|l|l|l|l|l|l|l|}
\hline & Sp initial & Et & mes & car & total conj. & sub.temp \\
\hline & & & & & & \\
\hline Artu & $10-\mathbf{5}$ & 30 & 5 & 33 & $68-\mathbf{3 4}$ & $9-\mathbf{4 . 5}$ \\
\hline Tristan & $52-\mathbf{1 5 . 7}$ & 32 & 11 & 31 & $74-\mathbf{2 2 . 4}$ & $150-\mathbf{4 5 . 4}$ \\
\hline Joinville & $15-\mathbf{6}$ & 31 & 1 & 10 & $42-\mathbf{1 7}$ & $33-\mathbf{1 3}$ \\
\hline QJM & $17-\mathbf{5 . 3}$ & 6 & 10 & 16 & $32-\mathbf{9 . 9}$ & $9-\mathbf{2 . 8}$ \\
\hline Froissart & $18-\mathbf{1 0 . 4}$ & 3 & 7 & 18 & $28-\mathbf{1 6 . 2}$ & $20-\mathbf{1 1 . 6}$ \\
\hline Monstrelet & $31-\mathbf{4 1 . 3}$ & 3 & 10 & 7 & $20-\mathbf{2 6 . 7}$ & $15-\mathbf{2 0}$ \\
\hline CNN & $26-\mathbf{1 5 . 6}$ & 13 & 6 & 4 & $23-\mathbf{1 3 . 8}$ & $20-\mathbf{1 2}$ \\
\hline Commynes & $40-\mathbf{4 2 . 1}$ & 1 & 11 & 19 & $31-\mathbf{3 2 . 6}$ & $6-\mathbf{6 . 3}$ \\
\hline Jehan & $15-\mathbf{1 0 . 6}$ & 11 & 6 & 18 & $35-\mathbf{2 4 . 6}$ & $32-\mathbf{2 2 . 5}$ \\
\hline
\end{tabular}

Tableau 2 : Répartition de quelques éléments initiaux dans les énoncés à sujet préverbal.

Il est difficile, à première vue, de déceler un évolution notable et des affinités régulières entre textes, hormis entre Joinville et Froissart. On observe en revanche des affinités ponctuelles, sur un trait précis.

En outre, hormis ces trois grandes tendances ( $\mathrm{Sp}$ ou conjonction ou subordonnée temporelle en tête), on observe un éparpillement assez grand des autres éléments initiaux, chacun d'eux n'excédant que rarement 5 occurrences au sein d'un même texte. Notons cependant une exception pour Tristan, texte dans lequel on rencontre 9 occurrences de subordonnée hypothétique :

(21) et se il la honte de ces deus chevaliers ne venge, ki sont si compaingnon de la Table Reonde, il se mesfait trop durement. (Tristan)

et 14 occurrences de constructions détachées (« topique en construction ») : 
(22) Li doi cevalier, ki dehors estoient et ki la bataille regardoient, quant il l'ont grant piece regardee, il enconmencent a parler entr'aus. (Tristan)

De tous les textes il se dégage néanmoins une tendance générale très nette : les quelques structures récurrentes que l'on observe lorsque le sujet est préverbal ne se rencontrent pas lorsqu'il est postverbal. Ainsi on ne trouve pas de conjonction seule suivie de VSp, et les rares occurrences de subordonnée temporelle sont toujours accompagnées d'un autre élément (adverbe ou SN, le plus souvent circonstanciel), ou bien, dans Tristan, elles expriment l'antériorité (3 occ), comme en (23) :

(23) Il dist a chiaus ki entour lui estoient: «Par Sainte Crois, il les metra tous quatre a desconfiture u a mort, et ançois que la nuis venist en ociroit il teus. X. com cist cevalier sont (Tristan)

Pour les « cas dispersés », la situation est comparable. Par exemple, alors que l'adverbe lors se trouve dans 4\% (12 occ.) des énoncés $\mathrm{SpV}$ de $Q J M$, il n'y en a aucune occurrence dans les énoncés VSp. De même, l'adverbe toutefois, que l'on rencontre à 4 reprises dans les énoncés $\mathrm{SpV}$ de Froissart, n'apparaît pas quand $\mathrm{Sp}$ est postverbal. Il est en revanche présent 4 fois, précédé de mais ou car, avec un sujet postverbal dans Tristan (et 1 fois dans $A r t u$ ), textes dans lesquels il n'apparaît pas avant SpV. Mais dans ces textes, en tête des énoncés $\mathrm{SpV}$, on a plusieurs occurrences de neporquant/nequedant ... absent des énoncés VSp.

On pourrait citer de nombreux autres exemples de la sorte, qui dénotent des phénomènes de répartition complémentaire, non pour l'ensemble des textes, mais au sein d'un même texte. Cette répartition se fait parfois de manière assez subtile ; ainsi un élément de même sémantisme peut être réalisé sous des formes différentes. C'est le cas pour les adverbiaux de cause. On rencontre ainsi des subordonnées causales en tête des énoncés $\mathrm{SpV}$ de plusieurs textes (Joinville, QJM, Froissart), mais pas en tête des énoncés à sujet postverbal. Dans ces derniers, on trouve en revanche, bien que rarement, des SN prépositionnels, généralement por ce. On rencontre une exception notable dans Tristan: alors que les subordonnées causales se trouvent en tête de certains énoncés VSp (24), d'autres débutent par por ce (25) :

(24) Et pour ce k'il voit bien et connoist tout apertement que de ses caus ne porroit il mie granment grever son anemi a ce que trop est de grant force, s'est il mis du tout au soufrir et se cuevre de son escu. (Tristan)

(25) car il l'avoit fait faire tout nouvelement ne n'i avoit nule entresaingne de son lignage. Pour ce nel reconnurent il pas a cele fois, mais il les reconnut mout bien, tout maintenant $\mathrm{k}^{\prime} \mathrm{il}$ vit lourescus... (id.)

Chez Froissart on rencontre un cas de subordonnée causale en tête d'un énoncé SpV (26), et un autre en tête d'un énoncé VSp (27):

(26) Et pour ce que il veoient que li rois et nous, vous volons aidier et porter a l'encontre de euls, il se refrenent de monstrer de fait leur mautalent (Froissart)

(27) ...et pour ce que ses fils nommés Edouwars n'eut point celle grasce ne bonne aventure d'armes, car tous ne sont pas ne ne pueent estre aourné de bonnes vertus, escei ils en haine et indignation de son peuple (Froissart)

Les énoncés (26) et (27) présentent cependant une différence. Si, dans les deux cas, le sujet de l'énoncé de $\mathrm{SpV}$ ou de VSp est le même que celui de la subordonnée causale qui précède, en revanche, dans le contexte immédiatement précédent ${ }^{15}$, on constate que le sujet de $\mathrm{SpV}$ y est mentionné, avec d'autres référents, tandis que celui de VSp ne l'est pas, l'énoncé étant centré sur le père d'Edouard : dans SpV (26) le sujet est déjà au centre de l'attention, dans VSp (27) il le devient.

Dans certains cas, on rencontre, au sein d'un texte, un même élément, mais, s'il est seul devant SpV il ne l'est pas devant VSp, ou inversement, ou bien encore il est accompagné d'un élément différent. Cela s'observe avec les subordonnées temporelles, mais aussi avec les subordonnées concessives ou hypothétiques : 
(28) ...et s'il chiet a la dame une espille, il l'amassera, car elle se pourroit affoler a soy baissier $(Q J M)$

(29) Et s'il le savoit bien davant qu'il fust marié, si l'a il oublié...(QJM)

Pour les subordonnées hypothétiques, la répartition est claire : en tête d'un énoncé VSp, la subordonnée est toujours suivie d'un adverbe à valeur d'opposition, souvent si (ou encore /toutefois), alors qu'elle ne l'est jamais en tête d'un énoncé $\mathrm{SpV}$. L'adverbe d'opposition souligne la valeur spécifique de l'enchaînement dénoté dans la proposition principale : en l'absence de $s i$, la conséquence découle naturellement de l'hypothèse/condition exprimée dans la subordonnée. Au contraire, dans les énoncés VSp, la conséquence est inattendue : elle s'oppose à l'enchaînement qu'aurait pu laisser prévoir la subordonnée, ce que souligne le sémantisme de si. Rétrospectivement, on peut considérer que la subordonnée a une valeur plutôt concessive ${ }^{16}$.

Les phénomènes de répartition complémentaire affectent aussi les compléments essentiels qui se trouvent en position initiale. Nous nous limiterons ici aux remarques concernant l'objet nominal. Rappelons tout d'abord que, dès le $13^{\text {ème }}$ siècle, l'ordre VO est devenu le plus fréquent, mais l'ordre OV n'a pas disparu pour autant, au moins au $13^{\text {ème }}$ siècle. Plus précisément, on rencontre deux schémas : l'ordre OVS, assez fréquent, et l'ordre OSV, beaucoup plus rare. L'ordre OVS a 2 valeurs principales : si l'objet est anaphorique, il a une valeur topicale, mais la construction est emphatique; si l'objet est indéfini ou intensifié, il s'agit d'un objet focal, nettement emphatisé. Dans Tristan, on rencontre parfois l'objet antéposé en tête d'un énoncé $\mathrm{SpV}$, mais il se trouve beaucoup plus souvent associé à un sujet postverbal (une bonne quinzaine de cas). Les objets sont en tout cas toujours anaphoriques, ou au moins liés au contexte précédent (par la présence d'un comparatif par exemple) :

(30) Et che li moustra il bien apertement a la fin de la bataille. (Tristan)

On rencontre 3 occurrences de ce type dans Artu, et 2 dans QJM :

(31) Si lui convient prendre en pacience, quar aultre remyde n'y peut il metre (QJM)

Dans Artu, Tristan et QJM, la situation est donc -presque- nette : l'objet préverbal apparaît avec un $\mathrm{Sp}$ postverbal. La situation est en revanche plus complexe dans Joinville et Froissart, puisqu'on trouve des occurrences d'objet préverbal aussi bien en tête de $\mathrm{SpV}$ que de VSp. Chez Joinville, dans les énoncés à sujet préverbal, l'objet a une valeur nettement emphatique, il correspond à un élément « nouveau », ou en tout cas inactif :

(32) Il geterent une planche a terre pour requeillir le roy et le conte d'Anjou, son frere, et mon seigneur Geffroi de Sergines et mon seigneur Phelippe de Annemos, et le marechal de France que en appeloit dou Meis, et le mestre de la Trinité et moy. Le conte de Poitiers il retindrent en prison jusques a tant que le roy leur eust fait paier les .iic. mille livres que il leur devoit faire paier...(Joinville)

Dans Froissart, au contraire, l'objet est anaphorique ou établit un lien par inférence avec le contexte précédent, à travers $n u l$ (pronominal) en (33) :

(33) La fu recordé et leu au lonch et tout hault li mesfait de messire Hue le Espensier ; ne a nuls des articles il n'oposa ne ne dist riens a l'encontre (Froissart)

La situation est pour ainsi dire inverse dans les énoncés VSp de ces deux mêmes textes : l'objet est anaphorique chez Joinville, et il correspond à un référent inactif chez Froissart :

(34) Chars avoient il assés, mais toutes aultres coses lor estoient si chieres et si court tenues, qu'il n'en pooient recouvrer (Froissart)

Les objets préverbaux se raréfient dans les textes plus tardifs: on ne rencontre que de très rares occurences de l'ordre OSpV dans CNN et Commynes, avec une valeur nettement emphatique, et il n'y a aucune occurrence d'objet initial dans Monstrelet et Jehan.

On observe donc, pour les objets nominaux, une situation analogue à celle des autres éléments initiaux : il n'y a pas d'association systématique de tel type d'objet à une position du sujet, mais, dans un même texte, 
une répartition complémentaire des objets entre les énoncés VSp et $\mathrm{SpV}$ en fonction de leur accessibilité cognitive. On voit donc que les phénomènes de répartition complémentaire des éléments initiaux entre les énoncés $\mathrm{SpV}$ et VSp constituent une constante, qui revêt néanmoins des modalités variables, donnant lieu à des « micro-systèmes " propres à chaque texte. De ce point de vue, il nous semble qu'il faut dépasser la simple opposition entre genres, qui n'en reste pas moins pertinente, comme l'ont bien montré Van Reenen et Schøsler (2000) dans leur étude contrastive de 7 particules (ainzi apres, donc, lors, or, puis, et si). Les auteurs montrent que les particules se comportent différemment entre elles, non seulement au regard du paramètre de la continuité/discontinuité thématique ${ }^{17}$, mais aussi selon les trois genres envisagées : textes littéraires, discours direct et chartes. Nous pensons que, au-delà des caractéristiques liées aux genres, se manifestent des pratiques pour ainsi dire idiolectales, dont il convient de dépasser la singularité pour dégager les propriétés plus générales propres à la « langue ».

Dans cette perspective, et pour revenir à notre propos, peut-on, pour les énoncés VSp, mettre au jour, comme nous l'avons fait pour les énoncés $\mathrm{SpV}$, quelques tendances en ce qui concerne les éléments initiaux, et la relation avec le contexte précédent?

\subsection{Les énoncés VSp : continuité et rupture}

Du point de vue de la forme ou du sémantisme des éléments initiaux, on ne constate pas de régularités communes à l'ensemble des textes. Mais, si l'on dépasse la forme même des éléments, ou leur sémantisme, on peut percevoir une évolution qui permet de mettre au jour une double caractéristique.

L'évolution au fil des textes est triple. On constate tout d'abord un recul de la proportion d'énoncés VSp qui débutent par un complément essentiel (objets direct et indirect, attribut) : ils représentent $23 \%$ de l'ensemble dans Artu, 22\% dans Tristan, 33\% dans Joinville, mais seulement 8\% dans QJM, $10 \%$ dans Froissart, 7\% dans $C N N$ (cela correspond à une seule occurrence), et il n'y en a aucune occurrence dans Monstrelet, Jehan et Commynes ${ }^{18}$. On observe par ailleurs un recul notable des éléments qui établissent un lien avec le contexte précédent, qu'il s'agissse de morphèmes démonstratifs (certains inclus dans les compléments essentiels), comme dans (35), (36) et (37) :

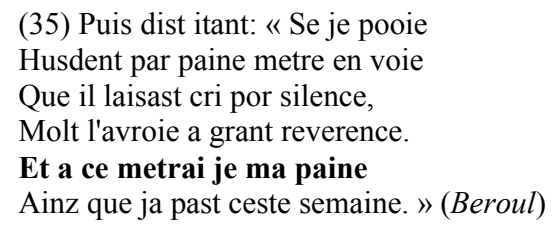

(36) Quant il vint desoz le chastel, il chevaucha si enbrons que a peinne le peüst l'en connoistre; et ce feisoit il por les chevaliers le roi qui de leanz issoient; (La mort Artu)

(37) Et sachiés que Plenorius avoit ja oïes nouveles que uns cevaliers errans estoit venus au pont, ki par sa proueche avoit ses deus freres menés dusques a outranche par force d'armes et venroit a lui combatre tout maintenant. Pour che s'estoit il bien apareilliés de ses armes...(Tristan en prose)

Enfin, les éléments servant à établir une comparaison, ou bien les adverbes spatio-temporels à caractère anaphorique (lors, leanz, la) connaissent eux aussi une forte baisse. De tels élements sont très fréquents dans Roland et Beroul, et encore dans Tristan et Joinville (58\% des cas pour ce dernier) :

\section{(38) Enz en voz bainz que Deus pur vos i fist, \\ La vuldrat il chrestïens devenir (Roland)}

alors qu'ils sont quasiment absents des textes plus tardifs ${ }^{19}$. Enfn, troisième caractéristique, on voit se développer dans ces textes plus tardifs des éléments à caractère logico-pragmatique, lesquels étaient beaucoup plus rares dans les textes précédents. Parmi eux, on observe la présence récurrente de subordonnées hypothétiques ou concessives suivies de si à valeur oppositive, ou bien de encore ou 
toutefois. Alors que ces éléments ne représentent que 5\% des cas dans Tristan et 10\% dans Froissart, ils deviennent plus fréquents par la suite : $25 \%$ des occurrences dans $Q J M, 29 \%$ dans Commynes (et le seul énoncé VSp de Montrelet commence par un tel élément). Voici un exemple :

\section{(39) ... et se il le portent et suesfrent un temps oultre lor volenté, si en rendent il en} la fin crueuls paiement (Froissart)

L'adverbe si apparaît régulièrement derrière ce type de subordonnées, mais on le rencontre aussi seul, souvent avec une valeur adversative/concessive, comme en (40), et aussi, plus tard, dans la tournure «si faire/être » qui marque une forme de conformité, et que l'on peut traduire par « l'être/le faire », comme en (41) :

(40) Chils rois ne faisoit compte de veoir la roine. Si estoit elle tres belle dame et feminine et doucement enlangagie (Froissart)

(41) Plust a Dieu qu'il voulsist tirer nostre chemin ». « Certes, sire », dit ung Anglois, « si fait il jusques a Bourdeaulx, comme il dit. » (Jehan)

Notons enfin la présence régulière, dans QJM, Froissart et $C N N$, des adverbes encore et ore :

(42) ... et li faites avoir son estat et vivre raisonnablement toute sa vie. Encores se pora il amender en consience, de qoi, tant c'a Dieu, il en vaudra grandement mieuls (Froissart)

(43) Le pouvre home court jour et nuit et quiert la robe dessus dite, et aultres chouses, dont a l'aventure il s' endebte grandement. Or est il bien venu $(Q J M)$

Cette liste n'est pas exhaustive, mais elle permet de dégager la double caractéristique commune de ces énoncés : continuité et rupture. Dans le cadre d'un enchaînement textuel, la première n'est pas très surprenante : qu'elle soit souvent associée à la seconde l'est en revanche davantage. Pour ce qui est de la continuité (discursive et/ou énonciative), elle se traduit par des marques de cohésion (expressions démonstratives, adverbes anaphoriques) mais aussi par des marques de connexité (conjonctions de coordination, adverbiaux logico-pragmatiques). Dans les textes les plus anciens, on rencontre surtout les premières, assez fréquentes dans Artu, Tristan et Joinville, alors que dans les textes plus tardifs, ce sont surtout les marques de connexité qui apparaissent.

Pour ce qui est des ruptures, elles sont de deux types, d'ordre syntaxique, et d'ordre logico-pragmatique. Les premières consistent en l'antéposition au verbe d'un complément essentiel, en particulier l'objet nominal, cas de figure bien représenté dans Artu, Tristan et Joinville. Il convient de noter que l'élément antéposé, qui provoque l'effet de rupture, est souvent aussi celui qui assure la continuité (présence d'un morphème démonstratif : voir 35-37 ci-dessus). Ces énoncés sont doublement marqués : par l'antéposition de l'objet nominal et par la postposition du sujet pornominal. Les ruptures d'ordre logicopragmatique (énoncés 39-43) présentent une apparente diversité, mais correspondent en fait à un même phénomène. Ainsi les subordonnées hypothétiques et concessives (39) relèvent toutes d'un même processus : la subordonnée laisse prévoir une certaine conclusion, qui n'est finalement pas celle qui advient; il se produit un retournement argumentatif. Dans les constructions en si/aussi faire (" le faire/faire de même/pareillement ») (41), l'adverbe traduit la conformité, qui concerne le procès lui-même. Associé à un verbe vicaire, si/aussi n'évoque aucun procès particulier, mais l'idée générale de procès, qui ne peut se préciser que par référence au contexte. Adverbe porteur de contingence, il « implique un débat au terme duquel une valeur est finalement retenue » (Guimier 1997). On a donc un débat qui entraîne la mise en balance - et donc en suspens - de la relation prédicative. Encore, dont la valeur se situe entre entre temporalité et quantité, exprime l'idée de surenchère, mais, surtout, celle d'un dépassement d'une frontière attendue (voir Fuchs 1994). Quant à ore, dont la valeur temporelle d'origine («maintenant») demeure, il exprime le seuil d'une nouvelle situation d'énonciation : ore instaure de nouvelles conditions d'énonciation, et marque ainsi une rupture (voir Ollier 1995).

Les retournements argumentatifs mettent en cause la force argumentative du premier élément d'un enchaînement : on valide un énoncé malgré un énoncé précédent qui aurait dû s'opposer à sa validation. Il y a renversement, et donc enchaînement inattendu. Ce processus est proche de celui qui se produit dans 
les cas de "mise en balance de la prédication ", où a lieu une mise en débat entre ce qui était censé advenir et ce qui advient réellement. On retrouve un processus analogue dans les énoncés en encore /ore et dans bon nombre des énoncés VSp de notre corpus. Peut-on, à partir de ces observations convergentes, proposer une interprétation de la postposition du pronom sujet ?

\subsection{L'inscription du locuteur dans son énoncé}

Nous venons de voir que l'inversion du suhet pronominal est associée à un enchaînement inattendu. C'est un constat qui s'inscrit tout à fait dans l'analyse du mode de donation référentielle du pronom " il » proposée par Kleiber : « $i l$ désigne un référent en continuité avec une situation manifeste dans laquelle le référent se trouve impliqué comme actant principal. » (Kleiber 1994 : 83). Or on peut considérer que mise en balance de la prédication, retournement argumentatif et enchaînement inattendu provoquent un " nonprolongement de la structure saillante », une " discontinuité avec la situation manifeste », et qu'il y a donc contradiction partielle avec le processus référentiel de $i l$ : la saillance référentielle est bien présente (d'où la présence du sujet pronominal), mais en revanche celle de la structure ne l'est pas (d'où une position inhabituelle du pronom, qui rend compte d'un dysfonctionnement). On a donc à la fois continuité référentielle et fonction nouvelle (par rapport à la fonction référentielle la plus courante du pronom).

Autrement dit, à une époque où la non-expression du sujet pronominal est encore courante, son expression dénote une insistance du locuteur, peu surprenante dans le cadre d'une mise en balance, d'un enchaînement inattendu : aussi surprenante que soit la relation prédicative énoncée, le locuteur la valide, et il souligne le fait qu'il la valide, en exprimant le pronom sujet, mais il indique en même temps son caractère problématique en recourant à une position inhabituelle et inattendue pour ce pronom sujet. Le sujet pronominal endosse ainsi une nouvelle fonction, celle de valider explicitement une relation problématique. On peut aussi considérer que ces énoncés traduisent une inscription de la subjectivité du locuteur, qui va se perdre avec la réduction des contextes d'occurrences des sujets postvervaux, perte que l'on peut considérer comme partie prenante du vaste mouvement du « subjectif » à l' « objectif » qui se produit ailleurs dans la langue à la même époque (voir Marchello-Nizia 1997).

A l'issue de ces observations, nous réinsisterons sur deux points : d'une part la présence de phénomènes de répartition complémentaire entre les énoncés VSp et $\mathrm{SpV}$, tant du point de vue des éléments initiaux que de la relation avec le contexte précédent, et d'autre part, pour les énoncés VSp, l'expression croissante d'une relation de rupture, souvent associée à une forme de continuité.

\section{Evolution de la syntaxe du sujet pronominal : des facteurs convergents}

Pour finir, nous aimerions tenter de replacer l'évolution de la syntaxe du sujet pronominal dans un contexte plus large. Rappelons tout d'abord quelques étapes décisives dans l'évolution de l'ordre des mots. A partir du $13^{\text {ème }}$ siècle, l'objet nominal tend à se fixer derrière le verbe (voir Marchello-Nizia 1995, Combettes 1988). C'est une évolution progressive, mais qui débute avant celle de la syntaxe du sujet. Plus tard, en moyen français, le verbe commence à reculer dans la proposition. Pour ce qui concerne le sujet, on observe, d'une part le developpement de son expression (dès l'ancien français) et, apparemment plus tard, la fixation de sa position, les deux évolutions étant progressives et se chevauchant. Il se produit d'autre part une clitisation du pronom. Question récurrente, il s'agit de savoir $s^{\prime} i l$ existe une corrélation entre ces différentes évolutions ${ }^{20}$. Il est à vrai dire difficile de répondre à cette question (les divergences de points de vue en témoignent), et seule l'étude d'un vaste corpus permettra d'affiner les chronologies respectives des trois évolutions, démarche d'autant plus nécessaire que l'on constate des fréquences très variables selon les textes, variation que l'on peut supposer en partie liée à leurs caractéristiques typologiques.

On peut néanmoins formuler quelques hypothèses. Il semble désormais acquis que, même s'ils ont certainement joué un rôle, ni la chute de la declinaison (et de la contrainte V2), ni la perte d'une morphologie riche (on trouve des discordances dès l'ancien français : $j$ 'iron) ne suffisent à expliquer le 
développement de l'expression du sujet et sa fixation en position préverbale. L'explication « informationnelle» (voir entre autres Vennemann (1976), Combettes (1988), Prévost (2001)) a permis de compléter cette approche : il y aurait eu un changement de principe organisateur de l'ordre des mots, depuis un principe pragmatique (informationnel) vers un principe syntaxique. En effet, dans la mesure où le sujet occupait souvent la position initiale, il s'est peu à peu fixé en cette place. On serait ainsi passé d'un ordre SXV (latin) à un ordre SVX en français moderne, via deux étapes intermédiaires : TXV puis TVX (aux $12^{\text {ème }}-13^{\text {ème }}$ siècles).

On peut cependant envisager d'autres explications, plus globales, qui ne remettent pas pour autant en cause les précédentes. Le développement des séquences $\mathrm{VO}^{21}$ pourrait être un point de départ aux autres évolutions : la fixation de l'objet nominal pourrait avoir causé celle du sujet, pour des raisons de clarté (nous admettons bien volontiers que l'érosion phonétique ne doit pas être exclue), mais aussi en raison d'une évolution plus générale. Du latin au français les syntagmes ont en effet subi ce que Marchello-Nizia (2003) appelle « une contrainte de contiguité ordonnée ». Certes celle-ci était déjà prévalente dans de nombreux textes latins (Lene Schøsler, communication personnelle), mais ce qui était une tendance forte va devenir une règle absolue. Or on constate qu'une contrainte analogue s'est développée au niveau de la phrase, dont les éléments connaissent un ordonnancement et une hiérarchisation croissants. Cette évolution pourrait être mise en relation avec une autre caractéristique du français, à savoir le développement d'une différenciation systématique entre déterminants et pronoms, pour les démonstratifs, les possessifs, et la plupart des indéfinis (voir Marchello-Nizia 2006 et Combettes $2006^{22}$ ). Les autres langues romanes n'ont pas poussé si loin une telle spécialisation, les catégories étant encore partiellement neutralisées. Dans la mesure où, dans les autres langues romanes, la syntaxe du sujet pronominal diffère largement de celle du français, on peut émettre l'hypothèse d'une corrélation entre ces différents faits.

Nous sommes consciente que les analyses présentées ici exigent d'être approfondies, ce que permettra la prise en compte d'un corpus plus large, qui devrait aussi faire apparaître des micro-évolutions spécifiquement liées aux caractéristiques typologiques des textes. Il ne s'agit pas de totalement remettre en cause les explications proposées jusqu'ici, tant en ce qui concerne l'inversion pronominale en ancien français que l'évolution de la syntaxe du pronom par la suite, mais plutôt d'apporter un éclairage nouveau et complémentaire, d'une part en proposant la prise en compte de facteurs sémantico-pragmatiques, d'autre part en essayant de corréler différemment l'évolution de la syntaxe du sujet pronominal à celle des autres changements qui se sont produits en français.

\section{Bibliographie}

\section{Textes étudiés :}

Beroul, Tristan, éd L. M. Defourques, Paris : Champion, 1947.

Chanson de Roland, éd. G. Moignet, Paris : Bordas, 1969.

La Mort le Roi Artu, éd. J. Frappier, Genève : Droz, 1954.

Tristan en prose, tome 1, éd. P. Ménard, Genève: Droz, 1987.

Jean de Joinville, La vie de Saint Louis, éd. J. Monfrin, Paris : Classiques Garnier, 1995.

Jean Froissart, Chroniques, éd. G. T. Diller, Genève : Droz, 1972.

Les XV Joyes du Mariage, éd. J. Rychner, Genève : Droz, 1963.

Enguerrand de Monstrelet, Chronique, éd. L. Douët d'Arcq, Paris : Société de l'Histoire de France (SHF), 1857-1862.

Les cent nouvelles nouvelles, éd. F. P. Sweester, Genève : Droz, 1966.

Le Roman de Jehan de Paris, éd. E. Wickersheimer, Paris : Champion (Librairie ancienne), 1923.

Philippe de Commynes, Mémoires, livre 1, éd. J. Calmette, tome 1, Paris : Belles Lettres (CHFM), 1964-65. 


\section{Bibliographie linguistique :}

Adams, M. (1987). Old French, Null Subjects and Verb Second Phenomena, Ph.D. Dissertation, University of California, Los Angeles.

Buridant, C. (2000) Grammaire nouvelle de l'ancien français. Paris : Sedes.

Combettes, B. (1988). Recherches sur l'ordre des éléments de la phrase en moyen français (Thèse pour le Doctorat d'Etat, Université de Nancy ; exemplaire dactylographié).

Combettes, B. (2006). Grammaticalisation et parties du discours : la différentiation des pronoms et des déterminants en français. In Guillot C., Heiden S. et Prévost S. (eds), A la quête du sens. Études littéraires, historiques et linguistiques en hommage à C. Marchello-Nizia, Lyon, ENS Editions

De Bakker, C. (1997). Germanic and Romance Inversion in French, a diachronic study, Leiden : Holland Institute of Generative Linguistics.

Dees, A. (1979). Variations temporelles et spatiales de l'ordre des mots en ancien et moyen français. In M. Wilmet (éd), Sémantique lexicale et sémantique grammaticale, Bruxelles: VUB Centrum voor TaalLiteratuuurwetenschap, 292-303.

Detges, U. (2003). Du sujet parlant au sujet grammatical . L'obligatorisation des pronoms sujets en ancien français dans une perspective pragmatique et comparative. Verbum, $X X V, 3,307-333$.

Dufresne, M. (1995). Etude diachronique de la cliticisation des pronoms sujets à partir du français médiéval. Revue Québécoise de Linguistique, 24, 84-109.

Foulet, L. (1930/1965). Petite syntaxe de l'ancien français. Paris : Champion.

Franzen, T. (1939). Etude sur la syntaxe des pronoms personnels sujets en ancien français, thèse pour le doctorat, Uppsala : Almquist et Wiksells.

Fuchs, C. (1994) Paraphrase et énonciation. Paris : Ophrys.

Fuchs, C. (2006). La place du sujet nominal en français. In Hrubaru F. et Velicu A. (éds) Enonciation et syntaxe, Cluj : Echinox, 9-25.

Guimier, C. (1997). La place du sujet clitique dans les énoncés avec adverbe initial. In C. Fuchs (éd.), La place du sujet en français contemporain, Louvain : Duculot (Coll. Champs linguistiques), 43-96.

Kleiber, G. (1994). Anaphores et Pronoms. Louvain la Neuve : Duculot.

Lahousse, K. (2003). La complexité de la notion de topique et l'inversion du sujet nominal. Travaux de Linguistique, 47, 111-136.

Marandin, J.-M. (2003). Inversion du sujet et structure de l'information dans les langues romanes. In D. Godard (éd), Langues romanes. Problèmes de la phrase simple, Paris : éditions du CNRS.

Marchello-Nizia, C. (1985). Dire le vrai. Genève : Droz.

Marchello-Nizia, C. (1995). L'évolution du français : ordre des mots, démonstratifs, accent tonique. Paris : Armand Colin.

Marchello-Nizia, C. (1997). Evolution de la langue et représentations sémantiques : du 'subjectif' à l'objectif' en français. In C. Fuchs et S. Robert (éds). Diversité des langues et représentations cognitives, Paris : Ophrys.

Marchello-Nizia, C. (2003). La "contrainte de contiguïé ordonnée" dans l'évolution du latin au français et aux autres langues romanes. In I. Uzcangar Vivar, E. Llamas Pombo et J.-M. Pérez Velasco (éds), Presencia y renovacion de la lingïistica francesa, Actes du Colloque des langues romanes de Salamanque, Salamanque : Ediciones Universidad, 231-244.

Marchello-Nizia, C. (2006). From personal deixis to spatial deixis: the semantic evolution of demonstratives from Latin to French. In M.Hickman et S. Robert (éds) Space in Languages: Linguistic Systems and Cognitive Categories, Amsterdam, Philadelphie : John Benjamins, pp. 103-120.

Martin, R. (1978). L'ordre des mots dans le Jehan de Saintré. In M. Wilmet (éd), Sémantique lexicale et sémantique grammaticale, Bruxelles : VUB Centrum voor Taal- Literatuuurwetenschap, 305-337.

Moignet, G. (1973). Grammaire de l'ancien français. Paris : Klincksieck. 
Ollier, M. -L. (1995). Or, opérateur de rupture. Linx, 32, 13-31.

Prévost, S. (1999). Aussi en position initiale : évolution sémantico-syntaxique du $12^{\text {ème }}$ au $16^{\text {ème }}$ siècle. Verbum, XXI, $3,351-380$.

Prévost, S. (2001). La postposition du sujet aux $15^{\text {ème }}$ et $16^{\text {ème }}$ siècles: approche sémantico-pragmatique. Paris : Editions du CNRS.

Prévost, S. (2002). Evolution de la syntaxe du pronom personnel sujet depuis le français médiéval : la disparition d'alternances signifiantes. In D. Lagorgette et P. Larrivée (éds) Représentations $d u$ sens linguistique, Munich : Lincom, Studies in Theoretical Linguistics, 22, 309-329.

Prévost, S. (à paraître). Expression et position du sujet pronominal en français. In Mémoires de la Société de Linguistique de Paris.

Price, G. (1966). Contribution à l'étude de la syntaxe des pronoms personnels en sujets en ancien français. Romania, $87,476-504$.

Rouveret, A. (2004). Les clitiques pronominaux et la périphérie gauche en ancien français. Bulletin de la Société de Linguistique de Paris, t. XCIX, fasc. 1, 181-237.

Rychner J. (1970) L'articulation des phrases narratives dans « La Mort Artu », Genève : Droz.

Schøsler, L. (1991). Les causes externes et internes des changements morpho-syntaxiques. Acta linguistica Hafniensia, 23, 83-112.

Skårup, P. (1975). Les premières zones de la proposition en ancien français. Essai de syntaxe de position. Etudes romanes de l'Université de Copenhague, Revue Romane, numéro spécial 6, Akademisk Forlag.

Vance, B. (1997). Syntactic Change in Medieval French : Verb-Second and Null Subjects, Dordrecht-BostonLondres : Kluwer Academic Publishers.

Van Reenen, P. et Schøsler L. (2000). The pragmatic functions of the Old French Particles AINZ, APRES, DONC, LORS, OR, PUIS, and SI. In S. C. Herring, P. van Reenen, et L. Schosler (éds) Textual Parameters in Older Languages, Amsterdam: Benjamins, 59-105.

Vennemann, T. (1976). Topics, subjects and word-order : from SXV to SVX via TVX. In J.M Anderson et C. Jones eds. Proceedings of the first international congress of Historical Linguistics. Amsterdam, 339-376.

Zink, G. (1997). Morpho-syntaxe du pronom personnel (non réfléchi) en moyen français $\left(14^{\text {ème }}-15^{\text {ème }}\right.$ siècles), Genève : Droz.

Zwanenburg, W. (1978). L'ordre des mots en français médiéval. In R. Martin (éd.), Etudes de syntaxe du moyen français, Paris : Klincksieck, 153-171.

\footnotetext{
${ }^{1}$ Voir Moignet (1973 : 359-361) pour une description des contextes d'occurrences de V1.

${ }^{2}$ La non-expression commence à reculer en moyen français et elle est devenue très rare au $17^{\text {ème }}$ siècle.

${ }^{3}$ Dans certains textes on observe toujours : subordonnée temporelle $+\mathrm{SpV}$. C'est le cas dans Tristan en prose : Quant il ont lour hiaumes ostés et il se sont entrebaisié, et entreconmandé a Nostre Signeur, ensi com cevalier errant doivent faire et faisoient a celui tans acoustumeement, il relacent lour hiaumes ..., tandis que dans d'autres textes on trouve toujours $s i$, comme dans la Conqueste de Constantinople de R. de Clari (début $13^{\text {ème }}$ ) : Quant li marchis vit que le kiertés fu si grans en le vile, et qu'il ne pooient avoir soulas ne confort de nule part, si manda tous chiaus de le vile de Genevois qu'il i avoit et uns et autres, si parla a aus et si leur dist.

${ }^{4}$ Elle est en partie développée dans Prévost S. (à paraître).

${ }^{5}$ Foulet postule cependant que, quand le verbe est en position initiale, le sujet non exprimé correspond à un pronom préverbal.

${ }^{6}$ Et du fait que le sujet pouvait être conjoint, $\mathrm{XSpV}$ est de toute façon compatible avec V2.
} 


\begin{abstract}
${ }^{7}$ La position de Vance (1997) mérite d'être précisée : elle considère que, du point de vue syntaxique, les sujets non exprimés («nuls ») équivalent à des sujet pronominaux postverbaux pendant la première période de l'ancien français, mais que les énoncés à pronom postverbal, par la suite, présentent des caractéristiques discursives qui les distinguent de ceux à sujet nul. Un changement syntaxique s'opèrerait à la fin de l'ancien français, et les sujets nuls en moyen français correspondraient à des pronoms préverbaux. L'étude de Vance s'appuie principalement sur deux textes, la Queste del Saint Graal (vers 1220) et Jehan de Saintré (1456). La prise en compte d'un corpus plus vaste nous a montré que les énoncés à sujets postverbaux ont toujours présenté des spécificités, comme la présente étude tente de le montrer.
\end{abstract}

${ }^{8}$ Par la suite, la limite de la zone verbale se déplacera, elle englobera les pronoms préverbaux (affaiblissement de ces derniers).

${ }^{9}$ Voire plus tard selon certains : les positions en matière de datation sont en effet assez divergentes. Selon Moignet (1973) et Adams (1987), qui s'appuient sur l'élision précoce de je, la cliticisation aurait débuté dès le $13^{\text {ème }}$ siècle. Adams met en outre en avant l'apparition, dès cette époque, de l'inversion complexe. Selon Dufresne (1995), cette dernière est encore rare, et elle considère que, à cette époque, les pronoms sont simplement rythmiquement faibles, ayant besoin de s'appuyer sur un autre mot (voir aussi Franzen 1939); à partir du $15^{\text {ème }}$ siècle ils deviennent purement fonctionnels, mais il faut attendre le siècle suivant pour que l'on puisse les considérer comme des clitiques (formant, selon Dufresne, un constituant syntaxique mais non phonologique). Notons cependant que l'on rencontre les première formes régimes en emploi sujet dès la fin du $12^{\text {ème }}$ siècle : « Moi et vos fumez en une hore engendré » (Ami Amile 1200). Faut-il y voir un premier signe d'une perte de prédicativité ? Assurément un tournant a lieu vers 1200, période à partir de laquelle la loi Tobler-Mussafia n'est plus valide : on peut désormais trouver des pronoms régimes atones en tête de phrase ; il n'est pas incongru de transposer cette possibilité aux pronoms sujets.

${ }^{10}$ Même s'il a été montré (Prévost 2001) que, à partir du milieu du $15^{\text {ème }}$ siècle, la seule explication informationnelle est insuffisante.

${ }^{11}$ La distinction entre le fonctionnement des $1^{\text {ère }}$ et $3^{\text {ème }}$ personnes mérite d'être approfondie, tant en ce qui concerne la position que l'expression du sujet. Prévost (à paraître) propose des éléments en ce qui concenre la position, et nous renvoyons, pour l'expression, aux hypothèses développées dans Detges (2003).

${ }^{12}$ Beroul et Roland n'atteignent pas les 35000 mots : ils comprennent respectivement 27000 et 29000 mots.

${ }^{13}$ La présente étude n'est évidemment pas pionnière, et elle s'appuie pour une part sur les travaux antérieurs (voir références dans la bibliographie), qu'ils correspondent à une approche globale (Foulet 1965, Price 1966, Skårup 1975, Martin 1979, Marchello-Nizia 1995, pour n'en citer que quelques-uns) ou se concentrent au contraire sur la présence de certains éléments initiaux (Van Reenen et Schøsler 2000 par exemple). Outre la présentation de données quantifiées assez précises et l'exploration de données textuelles en partie inédites, notre étude vise à mettre au jour les phénomènes de répartition complémentaire entre énoncés à sujets postverbaux et ceux à sujets préverbaux.

${ }^{14} \mathrm{Car}$, au moins en ancien français, s'apparente parfois davantage à un adverbe.

${ }^{15}$ p. 122 pour (26) et p. 41 pour (27).

${ }^{16}$ Pour une étude détaillée de $s i$, voir Marchello-Nizia 1985.

${ }^{17}$ Les auteurs tiennent compte de l'expression et de la position du sujet mais n'opèrent pas de distinction sytématique entre sujet pronominal et nominal. L'étude est en outre concentrée sur un type spécifique d'élément initial (certaines particules) tandis que nous envisageons ici tous les éléments initiaux.

${ }^{18}$ Notre corpus est néanmoins restreint : il faudra explorer d'autres textes pour confirmer cette disparition. Notons par ailleurs que les objets préverbaux se maintiennent davantage avec un sujet nominal.

${ }^{19}$ Il est un type d'éléments qui semble se raréfier plus tôt encore : il s'agit de compléments peu liés au contexte précédent, qui ne dénotent pas un effet de contraste fort, mais semblent opérer une simple mise en en relief de

l'élément initial X, comme dans l'énoncé suivant emprunté à Roland: Demi Espaigne vus durat il en fiet,/ L'altre meitet avrat Rollant, sis niés;

${ }^{20}$ Par exemple, selon Buridant (2000), la cliticisation aurait contribué à soustraire le sujet pronominal à la position postverbale (en faveur de la position préverbale) quand un autre élément occupait la position initiale. Cette 
explication est cependant discutable, puisqu'elle suppose la contrainte V2 encore active, et stricte, alors que ce n'est pas le cas : même en ancien français on rencontre des verbes en $3^{\text {ème }}$ position (voir $4 \mathrm{a}-\mathrm{d}$ ).

${ }^{21}$ D'un point de vue typologique, il s'agit de l'acquisition du dernier trait des langues « VO », les autres traits étant déjà acquis, en particulier les séquences déterminé-déterminant (voir Marchello-Nizia 1995).

${ }^{22}$ Combettes suggère que le phénomène peut en partie s'expliquer par la perte, pour le verbe, de la possibilité à fonctionner comme une démarcation entre syntagmes dès lors qu'il recule dans la phrase. 